\title{
El nivel de exposición a las estatinas no se asoció con la incidencia ni con la mortalidad por infarto agudo de miocardio: estudio ecológico
}

The level of exposure to statins was not associated with acute myocardial infarction incidence and mortality: ecological study

\section{Objetivos}

Evaluar si existe correlación* a nivel poblacional entre la incidencia de infarto agudo de miocardio (IAM) y su mortalidad, y la utilización de estatinas.

\section{Diseño y población}

Estudio ecológico* llevado a cabo en Suecia con datos obtenidos entre 1998 y 2002, que incluyó en 289 municipios a 1.926.113 hombres y 1.995 .981 mujeres de 40 a 79 años. La utilización de estatinas se calculó de las prescripciones realizadas y a partir de los registros de las farmacias y fue expresada en dosis diaria definida (DDD) por cada mil habitantes.

Para cada municipio se calculó la incidencia y la mortalidad anual por IAM y la tasa de revascularización coronaria en hombres y mujeres por grupo etario.

Se evaluó la correlación mediante análisis bivariado y multivariado. Se utilizó el tamaño poblacional del año 2000 y un índice de deprivación socioeconómica que incluía en su construcción información sobre los niveles educativos, salariales y de desocupación.
Nilsson y col. Journal of negative results in biomedicine 2011, 10:6.

\section{Resultados}

La tasa de utilización media de estatinas durante el periodo de cinco años estudiado aumentó unas tres veces (desde 46,2 hasta $131,1 \mathrm{DDD} / 1000$ habitantes por día en hombres y desde 28 hasta $87 \mathrm{DDI} / 1000$ habitantes por día en mujeres). Ver figura 1. En igual período la mortalidad por IAM en hombres sólo disminuyó de 2,2 a 1,72/1000 y en mujeres de 0.97 a 0.76 $/ 1000$.

La incidencia del IAM decreció en hombres de 8.37 a 7.81 /1000 y luego aumentó a 8.06 en 2002. En mujeres disminuyó los primeros tres años del estudio de 3,79 a 3,76 /1000 y luego aumentó a 3,82 en 2002.

No se observó asociación cruda entre el uso de estatinas y la mortalidad por IAM, ni tampoco luego de ajustar por edad, sexo, índice de deprivación económica, coordenadas geográficas y uso de fármacos antidiabéticos. Ver figura 1. El cambio en la utilización de estatinas entre 1998 y 2000 no mostró correlación con el cambio en la mortalidad por IAM entre 2000 y 2002.

Figura 1. Comparación temporal entre la incidencia y la mortalidad por infarto agudo de miocardio y el uso de estatinas en una población sueca de 40 a 79 años.

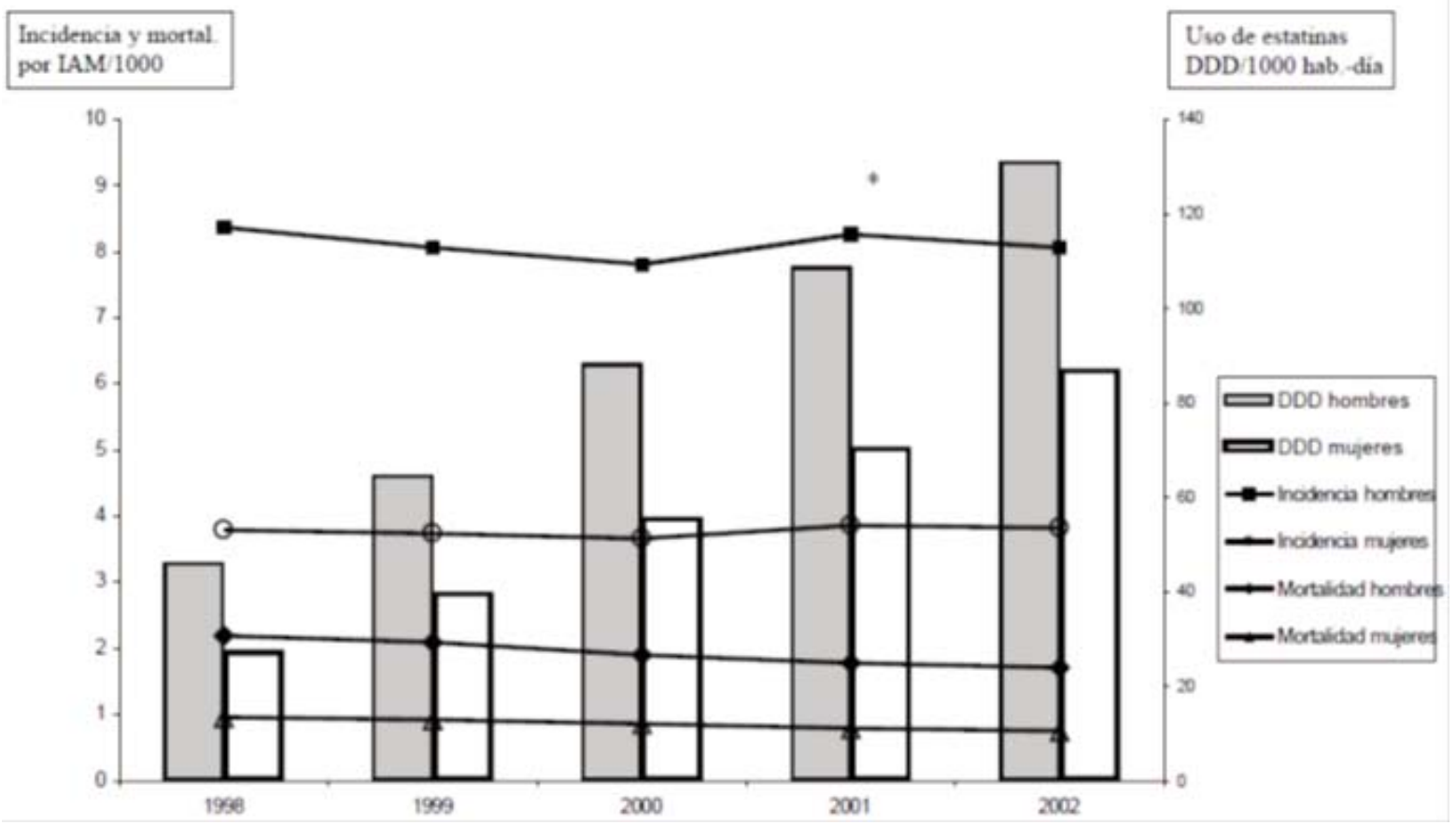

${ }^{*}$ Cambio en el punto de corte de los valores de troponina cardíaca T, troponina I o creatin kinasa (CK-MB) para hacer diagnóstico de infarto agudo de miocardio (IAM)

\section{Conclusión}

A pesar del uso extendido y del aumento en la utilización de estatinas, no pudo documentarse correlación entre su utilización y la incidencia o mortalidad por IAM en la población general.
Fuente de financiamiento: Health Research Council, Sweden y County Council of Östergötland. 


\section{Comentario}

A pesar de que gran parte de la población usaba estatinas en forma creciente, este trabajo no pudo documentar a nivel poblacional los beneficios que habían sido comunicado en los ensayos clínicos aleatorizados (ECCA). Sin embargo, debe recordarse que los ECCA son estudios metodológicamente mucho más protegidos de sesgos que los estudios ecológicos como el que acabamos de resumir, que no buscan asociación entre el uso de estatinas y resultados en un mismo paciente sino que lo hacen a nivel global. Una reciente revisión Cochrane de ECCA (celosamente resguardada de conflicto de intereses en la conducción de la revisión $y$, dentro de lo posible, respecto de los estudios primarios que fueron evaluados) concluyó que las estatinas reducen la mortalidad total, los eventos vasculares mayores y las revascularizaciones sin que se observe un exceso de efectos adversos en personas sin evidencia de enfermedad cardiovascular (prevención Primaria) ${ }^{1}$. Por lo tanto, es importante no sacar conclusiones apresuradas y descartar los potenciales beneficios de las estatinas (utilizadas apropiadamente).

Si bien entendemos y compartimos el entusiasmo por la prevención cuaternaria ${ }^{2,3,4}$, consideramos que no debemos hacernos eco de quienes afirmaron en forma ligera "...que este tipo de evidencia poblacional muestra que algunas veces los ensayos clínicos pueden arrojar resultados erróneos...". Esa afirmación, además de incorrecta en lo referente a las estatinas, supone que un diseño ecológico puede refutar los resultados de un ECCA.

Hecha esta aclaración, destaquemos al real valor del estudio y lo que debería alertarnos. Si bien los beneficios de las estatinas en términos relativos son bastante parecidos en todos los grupos de riesgo, el beneficio absoluto es mucho más marcado en los grupos de mayor riesgo.
En este sentido, es probable que las estatinas se estén administrando masivamente solo para modificar un valor del colesterol (resultado de la presión de laboratorios sobre pacientes y profesionales) y no para bajar el riesgo cardiovascular como recomiendan la mayoría de las guías, incluso las más terapéuticamente agresivas ${ }^{5}$. Esta masivización de su prescripción probablemente esté diluyendo sus beneficios a nivel poblacional.

Por otro lado, más allá de la efectividad de las estatinas, existen otros parámetros de ayuda para la toma de decisiones sanitarias a nivel global como lo son la costo-efectividad y el impacto presupuestario para un país, que deben ser analizados especialmente cuando está en discusión la asignación de los recursos públicos.

\section{Conclusiones de los comentadores}

Es de esperar que en pacientes con riesgo cardiovascular lo suficientemente alto (y más aún en prevención secundaria) el uso de estatinas impactará en beneficios poblacionales.

Sin embargo, consideramos que a nivel individual es pertinente valorar el riesgo cardiovascular individual de cada sujeto y luego establecer un proceso de toma de decisiones compartidas que incluya los siguientes tres elementos: 1) las opciones posibles (incluyendo la de no recibir tratamiento), 2) los resultados disponibles sobre la eficacia y la seguridad del uso de estatinas, 3) la probabilidad de ocurrencia de dichos resultados ${ }^{6}$.

Vale destacar que actualmente existen herramientas de ayuda para la toma de decisiones compartidas respecto de la prevención cardiovascular y que incluyen información global más allá de los valores de colesterolemia, que facilitan que nuestros pacientes puedan tomar decisiones acordes a sus valores y preferencias?

Agustín Ciapponi y Ana Herrera [ Servicio de Medicina Familiar y Comunitaria del Hospital Italiano de Buenos Aires. agustin.ciapponi@ hospitalitaliano.org.ar ]

Ciapponi A y Herrera A. El nivel de exposición a las estatinas no se asoció con la incidencia ni con la mortalidad por infarto agudo de miocardio: estudio ecológico. Evid Act Pract Ambul. 2014:17(4).Oct-Dic. 127-128. Comentado de: Nilsson S y col. No connection between the level of exposition to statins in the population and the incidence/mortality of acute myocardial infarction: An ecological study based on Sweden's municipalities. Journal of Negative Results in BioMedicine. 2011;10:6. PMID: 21609438.

\section{Referencias}

1. Taylor F y col. Statins for the primary prevention of cardiovascular disease. Cochrane Database of Systematic Reviews 2013, Issue 1. Art. No.: CD004816. DOI: 10.1002/14651858.CD004816.pub5

2. Gervás J. La prevención cuaternaria. OMC. 2004; 95:8

3. Gervás J. Moderación en la actividad médica preventiva y curativa Cuatro ejemplos de prevención cuaternaria en España. Disponible en file:///C:/AGUSTIN\%20en\%20N4e0n2/Evidencia/p3-5.pdf

4. Kopitowski K y col. Prevención cuaternaria: se pueden y se deben limitar los daños por la actividad sanitaria. Rev. Hosp. Ital. B.Aires 2013;33(3):90-5. Disponible en http://www.hospitalitaliano.org.ar/archivos/noticias attachs/47/documentos/15380 90-95-HI3-7 revision-C.pdf

5. Stone N y col. 2013 ACC/AHA Guideline on the Treatment of Blood Cholesterol to Reduce Atherosclerotic Cardiovascular Risk in Adults: A Report of the American College of Cardiology/American Heart Association Task Force on Practice Guidelines. Circulation. 2013

6. Ciapponi A. Toma de decisiones compartidas. Evid Act Pract Ambul. Ene-Mar 2012;15(1):2-4

7. Statin/Aspirin Choice. Decision Aid. Disponible en http://statindecisionaid.mayoclinic.org/index.php/statin/index (último acceso: 12/04/14). 